\title{
Microscopic models for fractionalized phases in strongly correlated systems
}

\author{
T. Senthil and O. Motrunich \\ Massachusetts Institute of Technology, 77 Massachusetts Ave., Cambridge, Massachusetts 02139
}

(Received 12 February 2002; published 19 November 2002)

\begin{abstract}
We construct explicit examples of microscopic models that stabilize a variety of fractionalized phases of strongly correlated systems in a spatial dimension larger than one, and in a zero external magnetic field. These include models of charge fractionalization in boson-only systems, and various kinds of spin-charge separation in electronic systems. We determine the excitation spectrum, and show the consistency with that expected from field theoretic descriptions of fractionalization. Our results are further substantiated by direct numerical calculation of the phase diagram of one of the models.
\end{abstract}

DOI: 10.1103/PhysRevB.66.205104 PACS number(s): 71.10. $-\mathrm{w}$, 05.50. $+\mathrm{q}$, 75.10.Jm, 75.10.Nr

\section{INTRODUCTION}

Considerable theoretical effort has gone into understanding the possibility of obtaining fractional quantum numbers for the excitations of strongly correlated systems in two or more spatial dimensions, and in weak or zero magnetic fields. Though much of the original interest arose in theories $^{1-3}$ of the high temperature superconductors, ideas based on fractionalization have since been proposed to account for the properties of a number of other poorly understood strongly correlated systems. ${ }^{4-9}$ Field theoretic methods have enabled enormous progress in obtaining a description of fractionalization. ${ }^{3,10-12}$ A number of exotic fractionalized phases have been accessed. The structure of the distinct possible excitations and the effective theory of their interactions ${ }^{3}$ has been elucidated in some detail. A crucial feature is the presence of discrete gapped vortexlike excitations-dubbed visons - apart from the excitations with fractional quantum numbers. It has become clear ${ }^{11,13}$ that fractionalized phases may be given a precise theoretical characterization through the notion of "topological order" - a concept elucidated clearly by Wen ${ }^{14}$ in work on the quantum Hall effect.

Scepticism has been voiced in some quarters over these developments due to the almost complete lack of microscopic models that can be shown to display the phenomena mentioned above. Specifically, consider a model of a manyparticle system with short-ranged interactions, and no special symmetries other than the global charge and/or spin conservation. Can fractionalization be shown to be obtained in such a model? Apart from its conceptual value, answering this question will also help to clarify the nature of the microscopic conditions that make it favorable for fractionalization to occur in a strongly correlated system.

There has been limited (though important) progress in a direct answer to this question. Numerical studies ${ }^{6}$ of a particular triangular lattice quantum spin-1/2 Heisenberg magnet with ring exchange interactions provide evidence of a state with a spin gap and a fourfold degenerate ground state on a torus as expected in a topologically ordered "spin liquid" with fractionalized "spinon" excitations. In the context of quantum dimer models, ${ }^{15}$ Moessner and Sondhi ${ }^{16}$ argued for a stable topologically ordered "liquid" phase on a triangular lattice. The standard interpretation of the dimer model views the dimers as caricatures of singlet bonds formed be- tween underlying Heisenberg spins on the lattice. From this point of view, the work of Ref. 16 provides supporting evidence, though not definitive proof, that models of Heisenberg antiferromagnets on triangular lattices do support a fractionalized spin liquid phase. However, as is well known, ${ }^{17}$ the quantum dimer model is exactly equivalent to a gauge theory-thus one may worry that establishing a topologically ordered phase in the dimer model still does not convince a skeptic that such phases can result in microscopic models with no special symmetries or a gauge structure.

Recently, Balents et al. ${ }^{18}$ argued that a particular easy axis quantum spin-1/2 model on a Kagomé lattice with short ranged (albeit complicated) interactions has a topologically ordered ground state with fractionalized excitations. This was done by reinterpreting it as a soluble point of the quantum dimer model on a triangular lattice but with three dimers rather than one dimer emerging from each site, and following the same arguments as in Ref. 16. (Also see Ref. 19 for a somewhat similar perspective.) However, some features of this model, such as the presence of two distinct visons, appear to be nongeneric (from the point of view of the effective field theory of fractionalized phases). This model also has an infinite number of local symmetries, and hence violates the requirement that fractionalization be demonstrated in models with no special symmetries. However, Balents et al. ${ }^{18}$ made the important observation that perturbing the model slightly to get rid of the local symmetries will preserve the fractionalized phase.

Finally, we note that a recent paper by Ioffe et al. ${ }^{20}$ proposed a physical realization of the triangular lattice quantum dimer model in its topologically ordered phase in a Josephson junction array. This too relies on the idea that small perturbations of the quantum dimer Hamiltonian (even if they destroy the microscopic gauge structure) do not destabilize the topologically ordered phase.

In this paper, inspired by these prior developments, we explicitly construct microscopic models that stabilize a wide variety of fractionalized phases. These include models for charge fractionalization in boson-only systems and various kinds of spin-charge separation in electronic systems. Our models involve only short-ranged interactions, and do not have any special symmetries other than global charge and/or spin conservation. We determine the excitation spectrum in the fractionalized phases, and explicitly show the consis- 
tency with that expected from the effective field theories. Our results complete the answer to the question of principle posed above, and will hopefully guide efforts to find materials and other even simpler models that realize these phases.

We begin by illustrating our construction with a simple Bose-Hubbard-type model of a system of bosons (with charge $q_{b}$ ) with short ranged interactions. The model has a global U(1) symmetry reflecting the conserved total boson number. We explicitly demonstrate the presence of two distinct Mott insulating phases in this model. In one, the excitations carry charges that are integer multiples of the underlying boson charge $q_{b}$. In the other, there are excitations with boson number $q_{b} / 2$, i.e., the bosons have fractionalized. This phase also has discrete $Z_{2}$ vortices, the visons, which are gapped. Upon tuning a parameter in the model, it is possible to drive transitions from either of the two Mott insulating phases to a superfluid phase. We further substantiate our arguments by performing a quantitative numerical calculation of the phase diagram of this model. The presence of topological order in one of the Mott insulating phases is detected numerically by the flux-trapping experiment discussed in Ref. 21 . We explicitly derive the effective field theory of the fractionalized phase and show that it is a theory of bosonic charge $q_{b} / 2$ fields coupled to a $Z_{2}$ gauge field in its deconfined phase.

Next we consider models of electrons coupled to superconducting phase fluctuations. These may be thought of as models of charge $e$ electrons interacting with spinless charge $2 e$ bosonic Cooper pairs. We show how the boson only models above may be extended to include coupling to electrons to provide a realization of various spin-charge separated phases. These models therefore provide explicit realizations of the routes explored in Refs. 12 and 3 for spin-charge separation. These spin-charge separated phases have spin-0 charge $e$ bosonic excitations (chargons or holons), spin-1/2 charge neutral fermionic spinons, and a gapped vison.

Of special interest are models that stabilize the nodal liquid $^{3,12}$ (alias $d_{x^{2}-y^{2}}$ RVB) phase-this has gapless fermionic nodal spinons, and has played an important role in theories of the cuprate materials. While recent experiments ${ }^{22,23}$ are not very encouraging on the possibility of fractionalization in the cuprates, it still is of theoretical interest to demonstrate models that realize the nodal liquid phase. Another theoretically controversial possibility is that of ordered magnetic phases that nevertheless are spin-charge separated. This was also first discussed ${ }^{12,13}$ in the context of cuprate physics, but is possibly relevant to a variety of other systems. We show how a model that stabilizes such ordered magnetic fractionalized phases may readily be obtained. This settles any doubts that may have been harbored on the possibility of such coexistence between magnetism and fractionalization. We then conclude with a brief discussion.

\section{FRACTIONALIZATION IN BOSON ONLY MODELS}

\section{A. Model and general arguments}

Consider a system of bosons on the "face-centered" square lattice in two dimensions shown in Fig. 1 modeled by the Hamiltonian

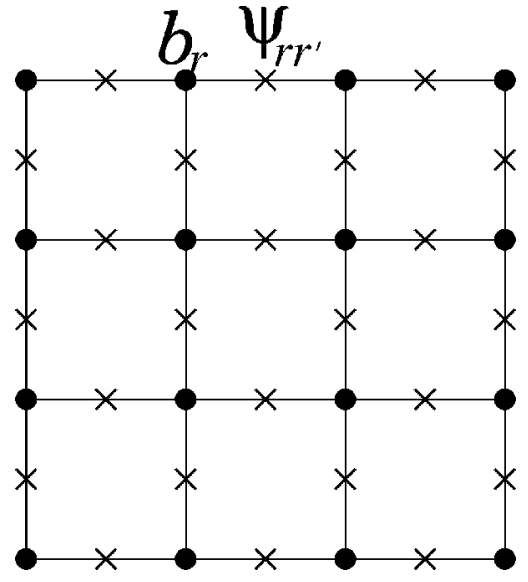

FIG. 1. Face-centered square lattice on which our model Eq. (1) is defined.

$$
\begin{gathered}
H=H_{w}+H_{\text {bond }}+H_{\text {ring }}+H_{u}, \\
H_{w}=-w \sum_{r, r^{\prime} \in r}\left(b_{r}^{\dagger} \Psi_{r r^{\prime}}+\text { H.c. }\right), \\
H_{\text {bond }}=-J_{\text {bond }} \sum_{\left\langle r r^{\prime}\right\rangle}\left[\left(\Psi_{r r^{\prime}}^{\dagger}\right)^{2}\left(b_{r} b_{r^{\prime}}\right)+\text { H.c. }\right], \\
H_{\text {ring }}=-K_{\text {ring }} \sum_{\square}\left(\Psi_{12}^{\dagger} \Psi_{23} \Psi_{34}^{\dagger} \Psi_{41}+\text { H.c. }\right), \\
H_{u}=u_{b} \sum_{r}\left(n_{r}^{b}\right)^{2}+u_{\psi} \sum_{\left\langle r r^{\prime}\right\rangle}\left(n_{r r^{\prime}}^{\psi}\right)^{2}+U \sum_{r} N_{r}^{2} .
\end{gathered}
$$

Here $b_{r}^{\dagger}=e^{i \theta_{r}}$ are bosons residing on the corner sites of the lattice, and $\Psi_{r r^{\prime}}^{\dagger}=e^{i \phi_{r r^{\prime}}}$ are bosons on the bond-centered sites, which we identify by the end points of the corresponding bond; $n_{r}^{b}$ and $n_{r r^{\prime}}^{\psi}$ are the corresponding boson numbers, $\left[\theta_{r}, n_{r^{\prime}}^{b}\right]=i \delta_{r r^{\prime}}$, and similarly for $\Psi_{r r^{\prime}}$ and $n_{r r^{\prime}}^{\psi}$. For technical convenience, we have chosen a rotor representation of the bosons (though this is not essential). The operator $N_{r}$ is defined through

$$
N_{r}=2 n_{r}^{b}+\sum_{r^{\prime} \in r} n_{r r^{\prime}}^{\psi} .
$$

The total boson number of the system is given by

$$
N_{\text {tot }}=\frac{1}{2} \sum_{r} N_{r} .
$$

The $w$ term is a boson hopping between the corner and the bond-centered sites, and $r^{\prime} \in r$ sums over all such bonds emanating from $r$. The term $K_{\text {ring }}$ is a ring exchange among four bond-centered sites belonging to the same square plaquette $\square$, while the term $J_{\text {bond }}$ is a similar ring-exchangelike boson interaction, but among three sites associated with a given bond $\left\langle r r^{\prime}\right\rangle$. The importance of ring exchange terms for promoting fractionalization is strongly suggested by the various field theoretic descriptions, ${ }^{3}$ and by previous studies 


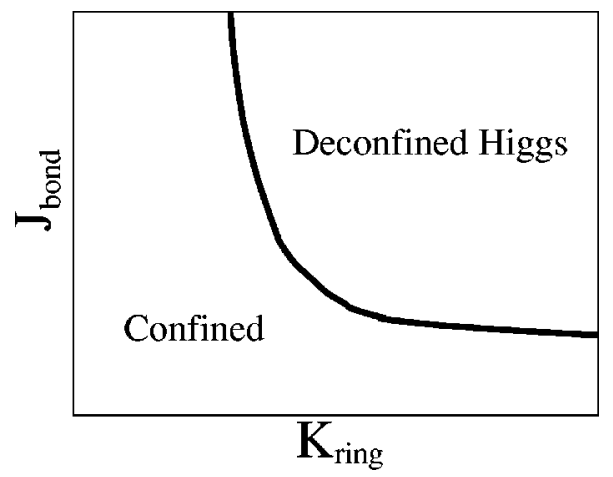

FIG. 2. Phase diagram of the $(2+1)$-dimensional compact QED coupled to a charge 2 scalar.

of microscopic models. ${ }^{6,18} u_{b}$ and $u_{\psi}$ are the usual on-site Hubbard terms. We have also included the Hubbard- $U$ term for the boson number $N_{r}$.

Despite the possibly unfamiliar form of the terms in the Hamiltonian, the following features are apparent. The model clearly has a global U(1) charge conservation symmetry associated with a global phase rotation of all the bosons. Note that if $b$ bosons are assigned a charge $q_{b}$, then the $\Psi$ bosons also have a charge $q_{b}$. There are no other special symmetries for general values of the parameters. In particular, there are no local symmetries. Furthermore, all the interactions are short ranged. We argue below that this model has a stable fractionalized insulating phase with charge $q_{b} / 2$ excitations and charge 0 visons above a ground state with no conventional broken symmetries.

Some gross features of the model can be guessed easily. At large $w$, the boson kinetic energy dominates and the system will be a superfluid. As $w$ is reduced, there will be a transition to an insulating phase. The nature of this insulating phase depends on the other parameters in the model. In particular, the insulator will be fractionalized for $J_{\text {bond }}, K_{\text {ring }}$, and $U$ large compared to $u_{b}$ and $u_{\psi}$. In the opposite limit, a conventional Mott insulator with charge quantized in units of $q_{b}$ will obtain.

To establish these results, it is useful to consider the special limit $w=0$; in this case, $\left[N_{r}, H\right]=0$ for every site $r$, and we can fix the value of $N_{r}$ for every $r$. Thus, in this limit, the model does have an infinite number of local symmetries. Later we will move away from this special limit, thereby destroying these local symmetries. For large $U$ at $w=0$, the ground state has $N_{r}=0$ everywhere. The $w=0$ model in the sector $N_{r}=0$ for every $r$ is readily understood as it can be regarded as the well-studied ${ }^{24} 3 \mathrm{D}$ compact $\mathrm{U}(1)$ gauge theory coupled to a charge 2 scalar field. Indeed, divide the underlying square lattice of Fig. 1 into $A$ and $B$ sublattices. Let $\theta_{r} \rightarrow \widetilde{\theta}_{r}=\epsilon_{r} \theta_{r}$ with

$$
\begin{aligned}
\epsilon_{r} & =+1 \quad \text { if } \quad r \in A \\
& =-1 \quad \text { if } \quad r \in B .
\end{aligned}
$$

To preserve the commutation relations, define the corresponding conjugate variables

$$
\widetilde{n}_{r}^{b}=\epsilon_{r} n_{r}^{b}
$$

Similarly, let $a_{r r^{\prime}}=\phi_{r r^{\prime}}$ if $r \in A, r^{\prime} \in B$ and $a_{r r^{\prime}}=-\phi_{r r^{\prime}}$ if $r \in B, r^{\prime} \in A$. Consider $a$ as a vector field $a_{r \alpha} \equiv a_{r, r+\hat{\alpha}}$, with $\hat{\alpha}=\hat{x}, \hat{y}$, and perform the corresponding transformation $n_{r r^{\prime}}^{\psi} \rightarrow E_{r \alpha}$ to the vector field $E_{r \alpha}$ conjugate to $a_{r \alpha}$. We have

$$
N_{r}=\epsilon_{r}\left(\boldsymbol{\Delta} \cdot \mathbf{E}+2 \tilde{n}_{r}^{b}\right) .
$$

The Hamiltonian then becomes

$$
\begin{aligned}
H= & -2 J_{\text {bond }} \sum_{r, \alpha} \cos \left(\Delta_{\alpha} \widetilde{\theta}_{r}+2 a_{r \alpha}\right)-2 K_{\text {ring }} \sum_{\square} \cos (\Delta \times \mathbf{a}) \\
& +u_{b} \sum_{r}\left(\tilde{n}_{r}^{b}\right)^{2}+u_{\psi} \sum_{r, \alpha}\left(E_{r \alpha}\right)^{2}
\end{aligned}
$$

while the constraint $N_{r}=0$ is simply the "Gauss law"

$$
\boldsymbol{\Delta} \cdot \mathbf{E}+2 \tilde{n}_{r}^{b}=0 .
$$

As promised, $H(w=0)$ is the same Hamiltonian as for the $(2+1)$-dimensional compact QED coupled to a charge 2 scalar. This permits us to take over the classic results of Fradkin and Shenker on this model which determined the phase diagram to be of the form shown in Fig. 2. In the "confined" phase, all excitations carrying "gauge charge" are confined. In the "deconfined Higgs" phase, static external objects with gauge charge 1 are not confined. Furthermore, there is a stable gapped $Z_{2}$ vortex (which we may identify with the vison). A number of different perspectives are available on these results. A useful physical one is to regard the deconfined Higgs phase as a "condensate" of the charge-2 scalar. Naively, such a condensate will have gapped vortices quantized in units of $\pi$. However, due to the compactness of the gauge field, space-time monopoles are allowed in the theory. These correspond to events where the vorticity changes by $2 \pi$-consequently the vortices acquire a $Z_{2}$ character.

It is also clear that the deconfined Higgs phase has a topological order: e.g., the ground state is fourfold degenerate on a torus. These are simply obtained by threading no or one vison through the two holes of the torus.

Consider now the excited states of the original Hamiltonian $H(w=0)$ for large but finite $U$. Consider states such that $N_{r_{0}}=1$ at some site $r_{0}$ and $N_{r}=0$ everywhere else. Such a state can be regarded as a static gauge charge +1 at $r_{0}$ (assuming $r_{0} \in A$ ). In the confined phase this sector costs an infinite energy in an infinite system. However, in the deconfined Higgs phase it costs only a finite energy. Remarkably, in the original boson model, such a state has a true electric charge of $q_{b} / 2$ [recall that $Q_{\text {tot }}=\left(q_{b} / 2\right) \Sigma_{r} N_{r}$ ]. Thus, in the deconfined Higgs phase, excitations with fractional quantum numbers for the true electric charge are allowed. In contrast, in the confined phase, finite energy excitations have gauge charge 0 -this requires that $\sum_{r \in A} N_{r}=\sum_{r \in B} N_{r}$. Consequently, the excitations carry true electric charge that are integer multiples of $q_{b}$, and hence are not fractionalized.

These results on the $w=0$ Hamiltonian thus follow as a straightforward application of the standard Fradkin-Shenker analysis of the phase diagram of gauge theories. However, 
they acquire even further importance here when we consider the Hamiltonian away from the $w=0$ limit when $H$ no longer has an infinite number of local symmetries. Consider a small $w$. This introduces fluctuations which mix states with different values of $N_{r}$ at the same site. However, for small $w$, these will not be capable of closing the gap to excitations about the ground state. Consider, in particular, the deconfined Higgs phase in the presence of a small $w$. The fractionally charged excitations are now allowed to hop from site to site and will acquire a kinetic energy of order $w$. However, they will survive as meaningful excitations. The other independent excitation, namely, the $Z_{2}$ vortex, will also survive the introduction of a small $w$. Thus the original model has, for nonzero but small $w$, a genuine fractionalized phase. (We can also add other more general boson hopping terms; clearly, the fractionalized phase will survive as long as these terms are weak.) In the subsequent subsections, we provide several direct confirmations of these arguments. In particular, we provide an explicit derivation of the effective field theory of the fractionalized phase and show that it is a theory of charge $q_{b} / 2$ chargons coupled to a $Z_{2}$ gauge field in its deconfined phase. This will also serve to make obvious our assertions on the properties of the model.

We emphasize that despite the ease with which this result has been obtained, it has enormous significance. The Hamiltonian for $w \neq 0$ has no special symmetries other than global charge conservation, and has only short ranged interactions. Nevertheless, it possesses a fractionalized phase with charge $q_{b} / 2$ excitations and a gapped vison consistent with that expected from earlier field theoretic descriptions of fractionalization.

\section{B. Numerical calculation of the phase diagram}

In this subsection, we substantiate our results by a direct numerical calculation of the phase diagram of the model. To that end, it is useful first to consider a path integral representation of the model. The Euclidean action may be written

$$
\begin{aligned}
S= & \epsilon \sum_{\tau}\left(H_{w}+H_{\text {bond }}+H_{\text {ring }}\right) \\
& -J^{\tau} \sum_{r \tau} \cos \left(\theta_{r \tau+1}-\theta_{r \tau}+2 \lambda_{r \tau}\right) \\
& -K^{\tau} \sum_{\left\langle r r^{\prime}\right\rangle \tau} \cos \left(\phi_{r r^{\prime}, \tau+1}-\phi_{r r^{\prime}, \tau}+\lambda_{r \tau}+\lambda_{r^{\prime} \tau}\right) \\
& -2 W^{\tau} \sum_{r \tau} \cos \left(\lambda_{r \tau}\right) .
\end{aligned}
$$

Here $\lambda_{r \tau} \in[0,2 \pi)$ is a phase variable living on the temporal links. To arrive at this form of the action, we first decoupled the Hubbard- $U$ term in the path integral and replaced all the Villain forms by cosines. The lattice spacing in the time direction is $\epsilon$, and the various couplings are $J^{\tau}=1 /\left(2 u_{b} \epsilon\right)$, $K^{\tau}=1 /\left(2 u_{\psi} \epsilon\right)$, and $W^{\tau}=1 /(4 U \epsilon)$.

The action represents a classical three-dimensional $X Y$ model with a global U(1) symmetry. As all the Boltzmann weights are positive, we may analyze the phase diagram of

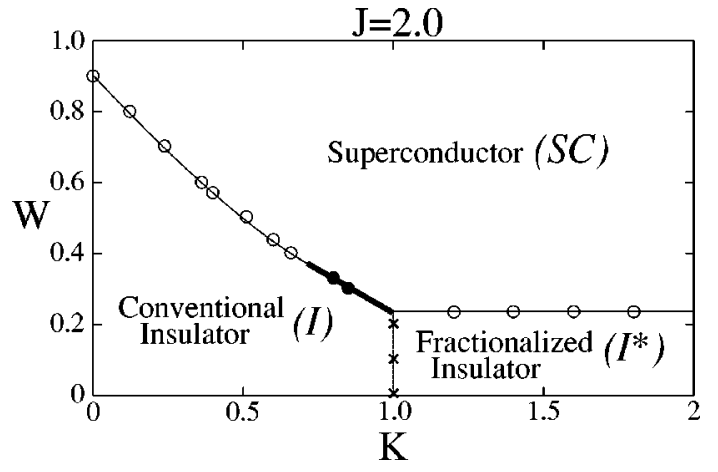

FIG. 3. Phase diagram of the classical model Eq. (10) at fixed $J=2.0$. We label the phases using the language of the original quantum model Eq. (1): Superconductor $S C$ is an $X Y$ ordered phase, while insulators $\mathcal{I}$ and $\mathcal{I}^{*}$ are two magnetically disordered but topologically distinct phases of the classical problem.

the model using direct Monte Carlo simulations. To avoid unimportant complications, we will consider a particular choice of coupling constants where $J^{\tau}=2 \epsilon J_{\text {bond }} \equiv J, K^{\tau}$ $=2 \epsilon K_{\text {ring }} \equiv K$, and $W^{\tau}=2 \epsilon w \equiv W$. Our choices of couplings $J, K$, and $W$ are such that the resulting classical statistical mechanical system is relatively isotropic in space-time. ${ }^{26}$

When $W=0$, the model is easily seen to reduce to the classical three-dimensional (3D) compact QED coupled to a charge 2 scalar. This has two phases, neither of which has $X Y$ order (which implies insulating behavior for the original quantum model), but which are topologically distinct. Turning on a small nonzero $W$ does not induce $X Y$ order, but preserves the topological distinction between the two phases. Upon increasing $W$, there is eventually a transition to an $X Y$ ordered phase. Thus we expect that a cut through the phase diagram in the $K-W$ plane for large but finite $J$ will look as in Fig. 3.

We verify this expectation by direct simulations of the classical model Eq. (10) on cubic lattices of sizes up to $12^{3}$ with periodic boundary conditions. We use heat-bath local updates and run over 5000 Monte Carlo iterations per each degree of freedom. We measure the $X Y$ order parameter in the original physical angles (e.g., $M=\Sigma_{j} e^{i \theta_{j}}$ ) and the superfluid stiffness $\rho_{s}$ associated with the direct boson hopping $w$ ( $\rho_{s}$ is defined in a standard way-see, e.g., Ref. 27). Both quantities can be used to identify transitions into the superfluid phase. We also measure the specific heat of the classical system; this serves as an unbiased indication of the thermodynamic phase transitions and their order. From these studies performed at fixed moderately large $J=2.0$, we obtain the phase diagram shown in Fig. 3, where we find three phases: an $X Y$ ordered phase $(S C)$ and two distinct disordered phases $\left(\mathcal{I}\right.$ and $\left.\mathcal{I}^{*}\right)$. We should point out one detail about our scans through the parameter space: To perform an accurate study of the $S C$ to $\mathcal{I}^{*}$ transition, we always start from a fully ordered state inside the $S C$ phase. We found that if we start from a completely disordered state in the $\mathcal{I}^{*}$ phase, the system often traps a vison and subsequently a vortex when going into the $S C$ phase (also see our discussion below), which significantly affects the measurements in our systems.

We analyze the transitions using finite-size scaling. The $\mathcal{I}$ 


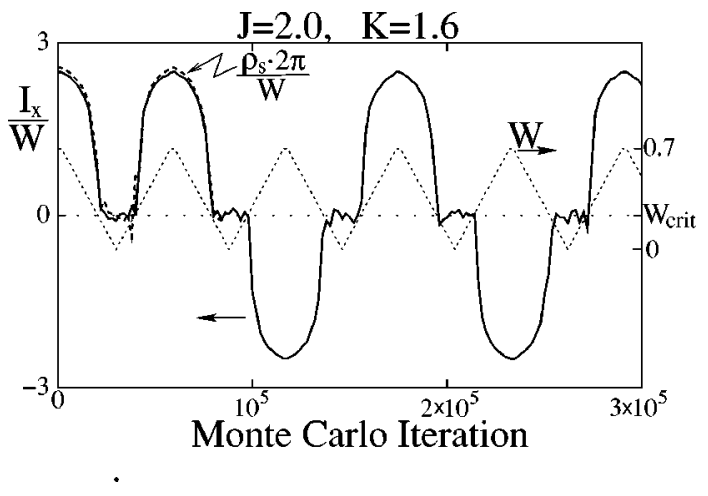

FIG. 4. Flux trapping experiment at $J=2.0$ and $K=1.6$. The system (of size $8^{3}$ ) is prepared with a single vortex in the annulus encircled by the $L_{x}$, deep in the $S C$ phase ( $W=0.7$; cf. Fig. 3 ). The vortex is detected by measuring the circulation $I_{x}$ of the superfluid current (solid line). The system is cycled between the $S C$ and $\mathcal{I}^{*}$ phases. The "sawtooth" dotted line is the Monte Carlo time variation of $W$ drawn so that the critical $W_{\text {crit }}$ coincides with the zero of $I_{x}$. For a trapped vortex, the magnitude of $I_{x}$ is set by the superfluid stiffness and is expected to be $\approx \rho_{s} 2 \pi$. The latter is shown with a dashed line for the first two cycles; the fact that the two quantities coincide indicates that the vortex remains trapped across the transition.

to $S C$ transition for small $K \leqslant 0.6$ and the $\mathcal{I}^{*}$ to $S C$ transition exhibit a 3D $X Y$ critical behavior; these are shown with open symbols in Fig. 3. For example, we can use the finite-size scaling relation, $\rho_{s} L=g\left(L^{1 / \nu} t\right)$, to locate the transitions and determine the correlation length exponent $\nu$. Using this standard procedure, we also observe an important distinction between the two disordered phases: The universal value $\left(\rho_{s} L\right)_{\text {crit }}$ at the $\mathcal{I}^{*}$ to $S C$ transition is found to be one-fourth that at the $\mathcal{I}$ to $S C$ transition, consistent with the charge fractionalization in the $\mathcal{I}^{*}$ phase.

The $\mathcal{I}$ to $S C$ transition for larger values of $K$ approaching the $\mathcal{I}^{*}$ phase, $0.7 \leqslant K \leqslant 1.0$, seems to be first order; this is indicated with filled symbols and a heavy line in the same figure. Our evidence for this is the observed strong sharpening of the specific heat peak for the larger systems, with the maximum value growing very strongly with the system size.

The $\mathcal{I}$ to $\mathcal{I}^{*}$ transition (marked by crosses in Fig. 3) is most easily identified by observing the specific heat. This is a true thermodynamic transition, but is not accompanied by any conventional ordering. Rather, it is associated with the onset of the topological order that characterizes the fractionalized phase. The universal properties of this transition may be described by a pure classical $3 \mathrm{D} Z_{2}$ gauge theory which in turn is dual to the global 3D Ising model. In our numerical calculations, the finite-size scaling of the specific heat peak is consistent with the $3 \mathrm{D}$ Ising universality class.

To illuminate the topological order in the $\mathcal{I}^{*}$ phase, we perform flux trapping "experiments" as described in Ref. 21 (also see Ref. 28). We summarize these experiments in Fig. 4. The system is prepared deep in the $S C$ phase with one vortex inside the annulus encircled by the periodic $L_{x}$; the physical angles $\theta$ and $\phi$ accumulate phase $2 \pi$ going around the $L_{x}$, and there is a superfluid current with circulation $I_{x}$ $\equiv \oint \vec{j} \cdot d \vec{l} \approx \rho_{s} 2 \pi$ in this direction. As we decrease $W$ toward the $\mathcal{I}^{*}$ phase, the vortex remains trapped all the way to the transition, and the magnitude of the superfluid current is set by $\rho_{s}$. In the $\mathcal{I}^{*}$ phase, the superfluid current is, of course, zero, but when we cycle the system back into the $S C$ phase, the superfluid current reappears with full initial strength but with a random sign. For comparison, if we create a double vortex and perform a similar $S C$ - $\mathcal{I}^{*}$ cycle (not shown), on similar time scales, the double vortex "tunnels out" before we reach the $\mathcal{I}^{*}$ phase and never reappears again. Similarly, a single or a double vortex created in the $S C$ phase both disappear when we approach the $\mathcal{I}$ phase and never reappear again upon subsequent $S C$ - $\mathcal{I}$ cycling.

In terms of the effective degrees of freedom of the $\mathcal{I}^{*}$ phase, the physical vortex is formed by a $\pi$ vortex in the chargon field and a vison. Bringing the system into the $\mathcal{I}^{*}$ phase, the vison remains gapped and is trapped in the annulus. Cycling the system back into the $S C$ phase, the vison binds a $\pi$ vortex in the chargon field, thus creating a physical vortex in the annulus but with a random sign.

\section{Effective field theory}

We now provide a mapping ${ }^{25}$ of the model Hamiltonian to a $Z_{2}$ gauge theory that will make obvious the results mentioned before. In addition, this yields an explicit derivation of the effective field theory for the fractionalized phase.

Consider the Hamiltonian Eq. (1). To bring out the possibility of a fractionalized phase, define the operators $b_{c r}^{\dagger}$ $=e^{i \theta_{c r}}$ and $\widetilde{\Psi}_{r r^{\prime}}^{\dagger}=e^{i \widetilde{\phi}_{r r^{\prime}}}$ through

$$
b_{c r}^{\dagger}=s_{r} e^{i \theta_{r} / 2}, \quad \widetilde{\Psi}_{r r^{\prime}}^{\dagger}=\Psi_{r r^{\prime}}^{\dagger} b_{c r} b_{c r^{\prime}} .
$$

Here $s_{r}= \pm 1$ so that $\theta_{c r} \in[0,2 \pi)$. The field $b_{c r}$ may be thought of as the "square root" of the operator $b_{r}$ and to carry a charge $q_{b} / 2$, and may be interpreted as a chargon operator. The field $\widetilde{\Psi}_{r r^{\prime}}$, on the other hand, is charge neutral.

Clearly, the boson number $N_{r}$ is conjugate to $\theta_{c r}$ and commutes with $\widetilde{\phi}_{r r^{\prime}}$, while $n_{r r^{\prime}}^{\psi}$ is conjugate to $\widetilde{\phi}_{r r^{\prime}}$ and commutes with $\theta_{c r}$ :

$$
\begin{gathered}
{\left[\theta_{c r}, N_{r}\right]=i, \quad\left[\widetilde{\phi}_{r r^{\prime}}, N_{r}\right]=0,} \\
{\left[\widetilde{\phi}_{r r^{\prime}}, n_{r r^{\prime}}^{\psi}\right]=i, \quad\left[\theta_{c r}, n_{r r^{\prime}}^{\psi}\right]=0 .}
\end{gathered}
$$

We can now write the Hamiltonian in terms of $\left(\theta_{c r}, N_{r}, \widetilde{\phi}_{r r^{\prime}}, n_{r r^{\prime}}^{\psi}\right)$, rather than the original variables. However, to recover the original physical Hilbert space, we need to impose the constraint

$$
e^{i \pi\left(N_{r}-\sum_{r^{\prime} \in r} n_{r r^{\prime}}^{\psi}\right)}=1
$$

this ensures that $n_{r}^{b}=\left(N_{r}-\sum_{r^{\prime} \in r} n_{r r^{\prime}}^{\psi}\right) / 2$ is an integer (original $b$-boson) number operator.

Making this (exact) change of variables, for the parts of the Hamiltonian Eq. (1) we obtain 


$$
\begin{gathered}
H_{w}=-w \sum_{r, r^{\prime} \in r}\left(b_{c r}^{\dagger} \widetilde{\Psi}_{r r^{\prime}} b_{c r^{\prime}}+\text { H.c. }\right), \\
H_{\text {bond }}=-J_{\text {bond }} \sum_{\left\langle r r^{\prime}\right\rangle}\left[\left(\widetilde{\Psi}_{r r^{\prime}}^{\dagger}\right)^{2}+\text { H.c. }\right], \\
H_{\text {ring }}=-K_{\text {ring }} \sum_{\square}\left(\widetilde{\Psi}_{12}^{\dagger} \widetilde{\Psi}_{23} \widetilde{\Psi}_{34}^{\dagger} \widetilde{\Psi}_{41}+\text { H.c. }\right) .
\end{gathered}
$$

Note that the $H_{\text {bond }}$ term acts as an Ising anisotropy on the $\widetilde{\Psi}_{r r^{\prime}}$ field. Considerable simplification is possible in the limit of large $J_{\text {bond }}$ and small $u_{b}$ to which we now specialize. The potential "seen" by the phase $\widetilde{\phi}_{r r}$ ' has two deep equivalent minima $\widetilde{\phi}_{r r^{\prime}}=0$ or $\pi$, which we label by $\sigma_{r r^{\prime}}^{z}=e^{i \widetilde{\phi}_{r r^{\prime}}}$ $= \pm 1$. The kinetic term $\left(n_{r r^{\prime}}^{\psi}\right)^{2}$ causes tunneling between the two wells. At each link, there are two low-energy states separated from all other states by a gap, leading to an effective two-state system. In the $\sigma_{r r^{\prime}}^{z}$ basis, we identify $e^{i \pi n_{r r^{\prime}}{ }^{\prime}}$ $=\sigma_{r r^{\prime}}^{x}$ since this operator translates $\widetilde{\phi}_{r r}$, by $\pi$. Also, the kinetic term $u_{\psi}\left(n_{r r^{\prime}}^{\psi}\right)^{2}$ is replaced by an effective transverse field $h \sigma_{r r^{\prime}}^{x}$. In this large $J_{\text {bond }}$ limit, the effective Hamiltonian becomes

$$
\begin{aligned}
H_{\mathrm{ch}}\left[b_{c}, \sigma\right]= & -2 w \sum_{\left\langle r r^{\prime}\right\rangle}\left(\sigma_{r r^{\prime}}^{z} b_{c r}^{\dagger} b_{c r^{\prime}}+\text { H.c. }\right)+U \sum_{r} N_{r}^{2} \\
& -2 K_{\text {ring }} \sum_{\square} \sigma_{12}^{z} \sigma_{23}^{z} \sigma_{34}^{z} \sigma_{41}^{z}-h \sum_{\left\langle r r^{\prime}\right\rangle} \sigma_{r r^{\prime}}^{x},
\end{aligned}
$$

while the constraint [Eq. (13)] is written as

$$
(-1)^{N_{r}} \prod_{r^{\prime} \in r} \sigma_{r r^{\prime}}^{x}=1
$$

at each site $r$.

This effective model is precisely the quantum problem of chargons coupled to a fluctuating $Z_{2}$ gauge field in two dimensions introduced and analyzed in Ref. 3. This model is known to have a phase diagram of the kind shown in Fig. 3. In particular, there is an insulating fractionalized phase where the chargon fields are deconfined and there is a gapped vison (which occurs for large $K_{\text {ring }}$, and small $w$ ).

\section{Generalization to arbitrary commensurate filling}

Our results are readily generalized to arbitrary commensurate values of the total average number of bosons per unit cell. Consider a modification of the Hamiltonian where the $U$ term is replaced by

$$
U\left(N_{r}-N_{0}\right)^{2}
$$

with $N_{0}$ a constant. At such commensurate density (rational values of $N_{0}$ ), insulating phases of the bosons will be possible. Again, in the limit of large $J_{\text {bond }}, K_{\text {ring }}$, and $U$ and small $u_{b}, u_{\psi}$, and $w$, this insulator will be fractionalized.
Conventional (i.e., nonfractionalized) insulating states are of course possible in other limits. All of these cases are readily studied using the methods of the Sec. II C. Indeed, a nonzero $N_{0}$ is trivially incorporated with no essential change leading to an effective Hamiltonian Eq. (17) but with the modified $U$ term above. As a special case of some interest, consider $N_{0}$ $=1$. The resulting model was previously suggested ${ }^{29,30}$ as an effective model of frustrated easy-plane spin-1/2 quantum antiferromagnets in two dimensions. A recent study by Park and Sachdev ${ }^{31}$ explicitly demonstrated the presence of the expected two insulating phases: a bond density wave crystal with confined excitations and a fractionalized phase. The fractionalized phase is more stable in this $N_{0}=1$ case due to additional frustration coming from the Berry phase terms.

\section{MODELS FOR SPIN-CHARGE SEPARATION}

In this section, we generalize the models of Sec. II to construct models that display spin-charge separated phases. We follow the route to spin-charge separation explored in Refs. 12 and 3 by considering models of electrons coupled to superconducting phase fluctuations. These may be thought of as models of spin-1/2 charge $e$ electrons interacting with spin-0 charge $2 e$ Cooper pairs. As shown below, the independent excitations of the spin-charge-separated phase are (i) a spin- 0 charge $e$ chargon, (ii) a spin- $1 / 2$ charge 0 spinon, and (iii) a spinless charge neutral $Z_{2}$ vortex-the vison. When either a chargon or spinon is taken all the way around a vison, the system acquires a phase of $\pi$. This structure is exactly what is expected on the basis of the effective field theories of stable spin-charge-separated phases. Indeed, as shown below, it is possible to provide an explicit derivation of the effective field theory as the correct description of our models in appropriate limits.

In the models presented below, the spinons are fermions while the chargons are bosons. An important property of the spinons is that their number is not conserved. There are "pairing" terms in the Hamiltonian describing the spinon dynamics. Different spin-charge-separated phases obtain based on the pairing symmetry of the spinons. Below we will discuss two different pairing symmetries as illustrative examples.

\section{A. Model for $d$-wave paired spinons}

Consider the following model:

$$
\begin{gathered}
H_{d \text { wave }}=H_{t}+H_{\Delta}+H_{w}+H_{\text {bond }}+H_{\text {ring }}+H_{u}, \\
H_{t}=-t \sum_{\left\langle r r^{\prime}\right\rangle}\left(c_{r \alpha}^{\dagger} c_{r^{\prime} \alpha}+\text { H.c. }\right), \\
H_{\Delta}=\sum_{\left\langle r r^{\prime}\right\rangle} \Delta_{r r^{\prime}}\left[\Psi_{r r^{\prime}}^{\dagger}\left(c_{r \uparrow} c_{r^{\prime} \downarrow}-c_{r \downarrow} c_{r^{\prime} \uparrow}\right)+\text { H.c. }\right], \\
H_{u}=u_{\psi} \sum_{\left\langle r r^{\prime}\right\rangle}\left(n_{r r^{\prime}}^{\psi}\right)^{2}+U \sum_{r}\left(N_{r}-N_{0}\right)^{2} .
\end{gathered}
$$


Here $c_{r \alpha}$ represents the destruction operator for an electron at site $r$ and spin $\alpha$. The electron is taken to have charge $e$. The operator $\Psi_{r r^{\prime}}$ may, in this model, be considered a Cooper pair living on the bonds of the lattice. In addition, these Cooper pairs on the bonds are coupled to other Cooper pair degrees of freedom $b_{r}$ residing on the sites of the lattice. The corresponding boson-only terms $H_{w}, H_{\text {bond }}$, and $H_{\text {ring }}$ are the same as before. The operator $N_{r}$ is defined through

$$
N_{r}=2 n_{r}^{b}+\sum_{r^{\prime} \in r} n_{r r^{\prime}}^{\psi}+\sum_{\alpha} c_{r \alpha}^{\dagger} c_{r \alpha} .
$$

Clearly, the total charge $Q_{\mathrm{tot}}=e \Sigma_{r} N_{r}$. The number $N_{0}$ is a constant that sets the average charge per site. We take the "pairing amplitude" $\Delta_{r r}$, to have a $d_{x^{2}-y^{2}}$ symmetry.

If $U$ is large, the system will be in an insulating phase (for commensurate density). The properties of this insulator depend on the values of the other parameters in the Hamiltonian. In particular, for large $J_{\text {bond }}$ and $K_{\text {ring }}$, we argue that the insulator will be spin charge separated. The spinons are fermionic and have $d_{x^{2}-y^{2}}$ pairing symmetry.

We proceed as before and define the chargon field $b_{c r}$ and the neutral field $\widetilde{\Psi}_{r r^{\prime}}$ through Eqs. (11). It will also be extremely convenient to define a spinon field $f_{r \alpha}$ through

$$
c_{r \alpha}=b_{c r} f_{r \alpha} .
$$

As before, the total charge associated with each site $N_{r}$ is conjugate to the chargon phase $\theta_{c r}$ and commutes with both $\widetilde{\Psi}_{r r^{\prime}}$ and $f_{r \alpha}$ :

$$
\left[\theta_{c r}, N_{r}\right]=i, \quad\left[\widetilde{\Psi}_{r r^{\prime}}, N_{r}\right]=\left[f_{r \alpha}, N_{r}\right]=0 .
$$

As expected, the $f_{r \alpha}$ fields are formally charge neutral. Equations (12) also continue to hold. We further have

$$
\left[f_{r \alpha}, n_{r r^{\prime}}^{\psi}\right]=0,
$$

and the equality $c_{r \alpha}^{\dagger} c_{r \alpha}=f_{r \alpha}^{\dagger} f_{r \alpha}$ We may work with the set of variables $\left(b_{c r}, N_{r}, \widetilde{\Psi}_{r r^{\prime}}, n_{r r^{\prime}}^{\psi}, f_{r \alpha}\right)$ instead of the original set $\left(b_{r}, n_{r}^{b}, \Psi_{r r^{\prime}}, n_{r r^{\prime}}^{\psi}, c_{r \alpha}\right)$. As with the boson-only models, this requires imposing a constraint on the Hilbert space, which now takes the form

$$
(-1)^{N_{r}-} \sum_{r^{\prime} \in r} n_{r r^{\prime}}^{\psi}-f_{r}^{\dagger} f_{r}=1 .
$$

Continuing with the same steps as in Sec. II, we find that in the large $J_{\text {bond }}$ limit, the Hamiltonian reduces to the following:

$$
\begin{gathered}
H=H_{t}+H_{\Delta}+H_{\mathrm{ch}}\left[b_{c}, \sigma\right], \\
H_{t}=-t \sum_{\left\langle r r^{\prime}\right\rangle}\left(f_{r}^{\dagger} f_{r^{\prime}} b_{c r}^{\dagger} b_{c r^{\prime}}+\text { H.c. }\right), \\
H_{\Delta}=\sum_{\left\langle r r^{\prime}\right\rangle} \Delta_{r r^{\prime}}\left[\sigma_{r r^{\prime}}^{z}\left(f_{r \uparrow} f_{r^{\prime} \downarrow}-f_{r \downarrow} f_{r^{\prime} \uparrow}\right)+\text { H.c. }\right] .
\end{gathered}
$$

$H_{\text {ch }}\left[b_{c}, \sigma\right]$ is the same as before [Eq. (17)]. The constraint reduces to

$$
(-1)^{N_{r}-f_{r}^{\dagger} f_{r}} \prod_{r^{\prime} \in r} \sigma_{r r^{\prime}}^{x}=1
$$

We now argue that for $N_{0}$ an integer, there is a stable spin-charge-separated phase. We first note that the Hamiltonian above describes a $Z_{2}$ gauge theory of spinons and chargons coupled to the $Z_{2}$ gauge field. As such, for large $K_{\text {ring }} \gg h$, its structure is almost identical to the effective theory of a spin-charge-separated phase of Ref. 3. The main difference is in the nature of the spinon hopping term (the term $H_{t}$ ) which seems to couple together the spinons and the chargons. However, this is readily seen to be an unimportant difference.

First, consider the limit of small $t$, $w$ (but $t \ll w$ ) at large repulsion $U$. In this limit, the chargons will lock into a Mott insulating phase (at integer $N_{0}$ ). At $t=w=0$, the chargon number will be fixed at $N_{0}$ per site. Going slightly away from this limit, we may treat both $H_{t}$ and the chargon hopping term in perturbation theory to eliminate virtual charge fluctuations. The result will be an effective Hamiltonian describing the spinon and gauge degrees of freedom. To second order, the generated terms take the form

$$
-\sum_{\left\langle r r^{\prime}\right\rangle} \frac{\hat{V}^{\dagger} \hat{V}+\hat{V} \hat{V}^{\dagger}}{2 U},
$$

with $\hat{V}=t f_{r}^{\dagger} f_{r^{\prime}}+2 w \sigma_{r r^{\prime}}^{z}$. Expanding, we obtain two nontrivial terms: the first is simply spinon hopping coupled to the $Z_{2}$ gauge field, while the second is a spinon four fermion interaction. The effective Hamiltonian then becomes

$$
\begin{gathered}
H=H_{\mathrm{sp}, t}+H_{\mathrm{sp}, \mathrm{int}}+H_{\Delta}+H_{\mathrm{IGT}}[\sigma], \\
H_{\mathrm{sp}, t}=-t_{\mathrm{sp}} \sum_{\left\langle r r^{\prime}\right\rangle} \sigma_{r r^{\prime}}^{z}\left(f_{r}^{\dagger} f_{r^{\prime}}+\text { H.c. }\right), \\
H_{\mathrm{sp}, \text { int }}=-\lambda \sum_{\left\langle r r^{\prime}\right\rangle}\left[\left(f_{r}^{\dagger} f_{r^{\prime}}\right)\left(f_{r^{\prime}}^{\dagger} f_{r}\right)+\left(f_{r^{\prime}}^{\dagger} f_{r}\right)\left(f_{r}^{\dagger} f_{r^{\prime}}\right)\right] .
\end{gathered}
$$

Here the spinon hopping $t_{\mathrm{sp}}=2 t w / U$ and the spinon interaction strength $\lambda=t^{2} /(2 U)$. Furthermore, in this large $U$ limit, the constraint simply reduces to

$$
(-1)^{f_{r}^{\dagger} f_{r}+N_{0}}=\prod_{r^{\prime} \in r} \sigma_{r r^{\prime}}^{x} .
$$

As a function of $K_{\text {ring, }}$, this Hamiltonian undergoes a deconfinement transition. In particular, for large $K_{\text {ring }}$, the fluctuations of the gauge field may be ignored and the spinons are free to propagate. The nature of the spinon dispersion is easily found by considering the limit $K_{\text {ring }}=\infty$. In this limit, we may set $\sigma_{r r^{\prime}}^{z}=+1$ on every bond. The quadratic part of the spinon Hamiltonian is then formally the same as that describing noninteracting quasiparticles in a $d_{x^{2}-y^{2}}$ superconductor, and therefore describes gapless nodal spinons. The spinon interaction is a formally irrelevant perturbation at this free spinon theory. As we are specifically in the limit that $t \ll w$, we have $\lambda \ll t_{\mathrm{sp}}$ - thus the interaction term may be safely 
ignored. Making $K_{\text {ring }}$ finite also only leads to irrelevant perturbations to the free spinon theory so that the long distance spin physics of the spin-charge-separated phase is described by nodal fermionic spinons.

The argument above considered the limit of large $U$ and $K_{\text {ring }}$ but small $t$ and $w$. It is also instructive to consider the limit $t, w \gg U$. In this limit, the chargons are expected to Bose condense leading to an ordinary $d_{x^{2}-y^{2}}$ superconductor. The long distance physics of this superconducting phase is readily captured by a continuum theory which keeps a continuum chargon phase field and the nodal spinons. The electron kinetic energy term $H_{t}$ is then readily written as a spinon kinetic energy modified by the usual "Doppler shift" term coupling the gradient of the phase (the superflow) to a bilinear in the spinons. Vortices are permitted in this phase and have flux quantized in multiples of $h c / 2 e$. In the large $K_{\text {ring }}$ limit, it is easy to see that the core energy of an $h c / 2 e$ vortex will include a contribution proportional to $K_{\text {ring }}$. On the other hand, the core energy of hc/e vortices does not diverge as $K_{\text {ring }}$ goes to infinity. Now consider decreasing $w$ to induce a transition to the insulator. At large $K_{\text {ring }}$, it is clear that this will occur due to proliferation of $h c / e$ vortices rather than due to $h c / 2 e$ vortices. Following the general arguments in Refs. 3, 12, we will obtain a spin-chargeseparated phase. Note that, as argued in Ref. 12, the Doppler shift term coupling the chargons and spinons is formally irrelevant, and one obtains a nodal liquid phase.

\section{B. Model for $s$-wave paired spinons}

It is straightforward to modify the model above to obtain one that stabilizes a spin-charge separated phase with $s$-wave paired fermionic spinons with a spin gap. We merely modify the pairing term above to

$$
H_{\Delta}=\Delta \sum_{r} b_{r}^{\dagger} c_{r \uparrow} c_{r \downarrow}+\text { H.c. }
$$

Proceeding exactly as above, it is easily established that for large $J_{\text {bond }}, U$, and $K_{\text {ring }}$, such a spin-charge-separated phase is indeed realized.

\section{Model for spin-charge-separated magnetically ordered phases}

The effective field theories for spin-charge-separated phases strongly suggest the theoretical possibility of spincharge separation coexisting with magnetic long range order in a quantum phase. In this subsection, we show how the models above may be readily generalized to stabilize such phases. Consider a system consisting of two layers and a Hamiltonian of the form

$$
H=H^{(1)}+H^{(2)}+H^{(12)} .
$$

Here $H^{(1)}$ and $H^{(2)}$ refer to parts of the Hamiltonian that depend only on the degrees of freedom residing in layers 1 and 2, respectively. The interactions between the two layers are contained in the term $H^{(12)}$. We assume that layer 1 consists of a square lattice of Heisenberg spins with $S=1 / 2$ described by the Heisenberg antiferromagnetic model

$$
H^{(1)}=J_{1} \sum_{\left\langle r r^{\prime}\right\rangle} \mathbf{S}_{1 r} \cdot \mathbf{S}_{1 r^{\prime}}
$$

We assume that layer 2 is described by the Hamiltonian $H_{d \text { wave }}$ in Eq. (20) above, and that the interaction between the two layers is given by

$$
H^{(12)}=J_{\perp} \sum_{r} \mathbf{S}_{1 r} \cdot\left(c_{2 r}^{\dagger} \boldsymbol{\sigma} c_{2 r}\right)
$$

with $J_{\perp} \ll J_{1}$. We assume that at $J_{\perp}=0$, the layer 2 is in its spin-charge-separated (and hence topologically ordered) phase. In this limit, layer 1 will order antiferromagnetically. Turning on a weak coupling $J_{\perp}$ will induce antiferromagnetic ordering in layer 2 , but cannot destroy the vison gap. Consequently, the full model Hamiltonian will be in a phase that has magnetic long range order but nevertheless is spincharge separated.

\section{SUMMARY}

In this paper, we have discussed several concrete examples of microscopic models in two spatial dimensions that display quantum phases with fractionalized excitations. These models possess no special symmetries other than those associated with global charge or spin conservation and also have only short ranged interactions, and thus confirm that fractionalization is a theoretically acceptable possibility for strongly interacting many particle systems in spatial dimensions larger than 1 . These models explicitly realize earlier field theoretic descriptions of fractionalization phenomena.

A number of generalizations of our results are possible. Our models are easily generalized to arbitrary spatial dimension, and provide concrete examples of fractionalized phases in any spatial dimension $d>1$. For spin-charge-separated phases of electronic systems, we have chosen to describe models with fermionic spinons and bosonic chargons. Following the ideas in Ref. 29, these are readily modified to construct spin-charge-separated phases with fermionic chargons and bosonic spinons (at least with easy plane spin anisotropy). An additional upshot of our results is the construction of topologically ordered classical 3D $X Y$ models. $^{26}$

Finally, we mention that quantum phases with topological order have also been suggested ${ }^{32}$ to be suitable states of interest to quantum computation. The topological structure naturally protects the system from decoherence. This very preliminary application ${ }^{19,20}$ may also benefit from the results in this paper.

\section{ACKNOWLEDGMENTS}

We thank Leon Balents, Matthew Fisher, and Subir Sachdev for useful conversations. This work was supported by the MRSEC program of the National Science Foundation under Grant No. DMR-9808941. 
${ }^{1}$ P. W. Anderson, Science 235, 1196 (1987).

${ }^{2}$ S. Kivelson, D. S. Rokhsar, and J. Sethna, Phys. Rev. B 35, 8865 (1987)

${ }^{3}$ T. Senthil and M. P. A. Fisher, Phys. Rev. B 62, 7850 (2000).

${ }^{4}$ R. Coldea, D. A. Tennant, A. M. Tsvelik, and Z. Tylczynski, Phys. Rev. Lett. 86, 1335 (2001).

${ }^{5}$ B. Bernu, L. Candido, and D. M. Ceperley, Phys. Rev. Lett. 86, 870 (2001).

${ }^{6}$ G. Misguich, C. Lhuillier, and B. Bernu, Phys. Rev. B 60, 1064 (1999); W. LiMing, G. Misguich, P. Sindzingre, and C. Lhuillier, Phys. Rev. B 62, 6372 (2000).

${ }^{7}$ S. Chakravarty, S. Kivelson, C. Nayak, and K. Voelker, Philos. Mag. B 79, 859 (1999).

${ }^{8}$ C. H. Chung, J. B. Marston, and R. H. McKenzie, J. Phys. C 13, 5159 (2001).

${ }^{9}$ C. H. Chung, J. B. Marston, and S. Sachdev, Phys. Rev. B 64, 134 407 (2001).

${ }^{10}$ For work on fractionalization in frustrated spin models, see N. Read and S. Sachdev, Phys. Rev. Lett. 66, 1773 (1991); S. Sachdev and N. Read, Int. J. Mod. Phys. B 5, 219 (1991).

${ }^{11}$ X. G. Wen, Phys. Rev. B 44, 2664 (1991).

${ }^{12}$ L. Balents, M. P. A. Fisher, and C. Nayak, Phys. Rev. B 60, 1654 (1999); ibid. 61, 6307 (2000).

${ }^{13}$ T. Senthil and M. P. A. Fisher, Phys. Rev. B 63, 134521 (2001).

${ }^{14}$ X. G. Wen, Int. J. Mod. Phys. B 4, 239 (1990).

${ }^{15}$ D. S. Rokhsar and S. A. Kivelson, Phys. Rev. Lett. 61, 2376 (1988).

${ }^{16}$ R. Moessner and S. L. Sondhi, Phys. Rev. Lett. 86, 1881 (2001).

${ }^{17}$ E. Fradkin and S. A. Kivelson, Mod. Phys. Lett. B 4, 225 (1990); S. Sachdev and M. Vojta, J. Phys. Soc. Jpn. 69, 1 (2000); R. Moessner, S. L. Sondhi, and E. Fradkin, Phys. Rev. B 65, 024 504 (2002).

${ }^{18}$ L. Balents, M. P. A. Fisher, and S. M. Girvin, Phys. Rev. B 65, 224412 (2002)

${ }^{19}$ C. Nayak and K. Shtengel, Phys. Rev. B 64, 064422 (2001).

${ }^{20}$ L. B. Ioffe, M. V. Feigel'man, A. Ioselevich, D. Ivanov, M. Troyer, and G. Blatter, Nature (London) 415, 503 (2002).

${ }^{21}$ T. Senthil and Matthew P. A. Fisher, Phys. Rev. Lett. 86, 292 (2001).
${ }^{22}$ D. A. Bonn, Janice C. Wynn, Brian W. Gardner, Yu-Ju Lin, Ruixing Liang, W. N. Hardy, J. R. Kirtley, and K. A. Moler, Nature (London) 414, 887 (2001).

${ }^{23}$ J. C. Wynn, D. A. Bonn, B. W. Gardner, Yu-Ju Lin, Ruixing Liang, W. N. Hardy, J. R. Kirtley, and K. A. Moler, Phys. Rev. Lett. 87, 197002 (2001).

${ }^{24}$ E. Fradkin and S. H. Shenker, Phys. Rev. D 19, 3682 (1979).

${ }^{25}$ Our approach is an adaptation of that used in Ref. 24 to analyze gauge theories at large Higgs coupling.

${ }^{26}$ Note that the $W^{\tau}$ term in action (10) suppresses the fields $\lambda_{r \tau}$ and thus promotes the correlations of the physical angles $\theta_{r \tau}$ and $\phi_{r r^{\prime} \tau}$ in the time direction. In this sense, it is similar to the spatial boson hopping term $w$. Here we also mention a particularly simple classical model that is qualitatively very similar (but not identical) to the one described by the action (10). This threedimensional isotropic model has angles $\theta_{i}$ and $\phi_{i j}$ living on the corner and bond-centered sites of a cubic lattice, with the classical energy

$$
\begin{gathered}
-W \sum_{i, j \in i} \cos \left(\theta_{i}-\phi_{i j}\right)-J \sum_{\langle i j\rangle} \cos \left(\theta_{i}+\theta_{j}-2 \phi_{i j}\right) \\
-K \sum_{\square} \cos \left(\phi_{12}-\phi_{23}+\phi_{34}-\phi_{41}\right) .
\end{gathered}
$$

It is remarkable that this simple $X Y$-like model has two magnetically disordered phases, one of which is topologically ordered. In the main text, we have chosen to simulate a somewhat more complicated looking action (10) so as not to divert the reader's attention from the quantum Hamiltonian equation (1).

${ }^{27}$ M. Cha, M. P. A. Fisher, S. M. Girvin, M. Wallin, and A. P. Young, Phys. Rev. B 44, 6883 (1991); Y. H. Li and S. Teitel, ibid. 40, 9122 (1989).

${ }^{28}$ R. D. Sedgewick, D. J. Scalapino, and R. L. Sugar, Phys. Rev. B 65, 054508 (2002).

${ }^{29}$ E. Demler, C. Nayak, H.-Y. Kee, Y. B. Kim, and T. Senthil, Phys. Rev. B 65, 155103 (2002).

${ }^{30}$ S. Sachdev and K. Park, Ann. Phys. (N.Y.) 298, 58 (2002).

${ }^{31}$ K. Park and S. Sachdev, Phys. Rev. B 65, 220405 (2002).

${ }^{32}$ A. Kitaev, quant-ph/9707021 (unpublished). 